\title{
Coupling News Sentiment with Web Browsing Data Improves Prediction of Intra-Day Price Dynamics
}

\author{
Gabriele Ranco ${ }^{1}$ *, llaria Bordino ${ }^{2}$, Giacomo Bormetti ${ }^{3,4}$, Guido Caldarelli ${ }^{1,5,6}$, \\ Fabrizio Lillo ${ }^{3,4}$, Michele Treccani ${ }^{4,7}$
}

1 IMT Institute for Advanced Studies, Piazza San Francesco 19, 55100 Lucca, Italy, 2 Yahoo Labs, Barcelona, Spain, 3 Scuola Normale Superiore, Piazza dei Cavalieri 7, 56126 Pisa, Italy, 4 QUANTLab, Via Pietrasantina 123, 56122 Pisa, Italy, 5 ISC-CNR, Via dei Taurini 19, 00185 Roma, Italy, 6 London Institute for Mathematical Science, South St. 35 Mayfair, London W1K 2XF, United Kingdom, 7 Mediobanca S.p.A, Piazzetta E. Cuccia 1, 20121 Milano, Italy

*gabriele.ranco@gmail.com

\section{Abstract}

The new digital revolution of big data is deeply changing our capability of understanding society and forecasting the outcome of many social and economic systems. Unfortunately, information can be very heterogeneous in the importance, relevance, and surprise it conveys, affecting severely the predictive power of semantic and statistical methods. Here we show that the aggregation of web users' behavior can be elicited to overcome this problem in a hard to predict complex system, namely the financial market. Specifically, our in-sample analysis shows that the combined use of sentiment analysis of news and browsing activity of users of Yahoo! Finance greatly helps forecasting intra-day and daily price changes of a set of 100 highly capitalized US stocks traded in the period 2012-2013. Sentiment analysis or browsing activity when taken alone have very small or no predictive power. Conversely, when considering a news signa/ where in a given time interval we compute the average sentiment of the clicked news, weighted by the number of clicks, we show that for nearly $50 \%$ of the companies such signal Granger-causes hourly price returns. Our result indicates a "wisdom-of-the-crowd" effect that allows to exploit users' activity to identify and weigh properly the relevant and surprising news, enhancing considerably the forecasting power of the news sentiment.

\section{Introduction}

The recent technological revolution with widespread presence of computers, users and media connected by Internet has created an unprecedented situation of data deluge, changing dramatically the way in which we look at social and economic sciences. As people increasingly use the Internet for information such as business or political news, online activity has become a mirror of the collective consciousness, reflecting the interests, concerns, and intentions of the global 
Reduction Agency, grant HDTRA1-11-1-0048; Progetto di Interesse CNR CrisisLab; and EUROPEAN COMMISSION CRISIS-ICT-2011288501. The authors declare that Michele Treccani is working for Mediobanca S.p.A. and llaria Bordino is working for Yahoo! Labs. The authors also declare that QUANTLab is not a commercial affiliation. Mediobanca S.p.A. and Yahoo! Labs provided support in the form of salaries for authors IB and MT but did not have any additional role in the study design, data collection and analysis, decision to publish, or preparation of the manuscript. The specific roles of these authors are articulated in the "author contributions" section.

Competing Interests: The authors declare that Michele Treccani is working for Mediobanca S.p.A. and llaria Bordino is working for Yahoo! Labs. The authors also declare that QUANTLab is not a commercial affiliation. These affiliations do not alter the authors' adherence to PLOS ONE policies on sharing data and materials. population with respect to various economic, political, and cultural phenomena. Humans interactions with technological systems are generating massive datasets documenting collective behaviour in a previously unimaginable fashion $[1,2]$. By properly dealing with such data collections, for instance representing them by means of network structures $[3,4]$, it is possible to extract relevant information about the evolution of the systems considered (i.e. trading [5], disease spreading [6,7], political elections [8]).

A particularly interesting case of study is that of the financial markets. Markets can be seen as collective decision making systems, where exogenous (news) as well as endogenous (price movements) signals convey valuable information on the value of a company. Investors continuously monitor these signals in the attempt of forecasting future price movements. Because of their trading based on these signals, the information is incorporated into prices, as postulated by the Efficient Market Hypothesis [9]. Therefore the flow of news and data on the activity of investors can be used to forecast price movements. The literature on the relation between news and price movement is quite old and vast. In order to correlate news and price returns one needs to assess whether the former is conveying positive or negative information about a company, a particular sector or on the whole market. This is typically done with the sentiment analysis, often performed with dedicated semantic algorithms as described and reviewed in the Methods Section.

In this paper, we combine the information coming from the sentiment conveyed by public news with the browsing activity of the users of a finance specialized portal to forecast price returns at daily and intra-day time scale. To this aim we leverage a unique dataset consisting of a fragment of the log of Yahoo! Finance, containing the news articles displayed on the web site and the respective number of "clicks", i.e. the visualizations made by the users. Our analysis considers 100 highly capitalized US stocks in a one-year period between 2012 and 2013.

For each of these companies we build a signed time series of the sentiment expressed in the related news. The sentiment expressed in each article mentioning a company is weighted by the number of views of the article. In our dataset each click action is associated with a timestamp recording the exact point in time when such action took place. Thus we are able to construct time series at the time resolution of the minute. To the best of our knowledge, this is the first time that an analysis like the one described in this paper is conducted at such intra-day granularity. The main idea behind this approach is that the sentiment analysis gives information on the news, while the browsing volume enable us to properly weigh news according to the attention received from the users.

We find that news on the same company are extremely heterogeneous in the number of clicks they receive, an indication of the huge difference in their importance and the interest these news generate on users. For $70 \%$ of the companies examined, there is a significant correlation between the browsing volumes of financial news related to the company, and its traded volumes or absolute price returns. More important, we show that for more than $50 \%$ of the companies (at hourly time scale), and for almost $40 \%$ (at daily time scale), the click weighted average sentiment time series Granger-cause price returns, indicating a rather large degree of predictability.

\section{Data \\ Stocks considered}

Our analysis is conducted on highly capitalized stocks belonging to the Russell 3000 Index traded in the US equity markets, which we monitor for a period of one year between 2012 and 2013. Among all companies, we selected the 100 stocks with the largest number of news published onYahoo! Finance during the investigated period. The ticker list of the investigated 
stocks with a distinctive numerical company identifier follows: $1 \mathrm{KBH}, 2 \mathrm{LEN}, 3 \mathrm{COST}, 4$ DTV, 5 AMGN, 6 YUM, 7 UPS, 8 V, 9 AET, 10 GRPN, 11 ZNGA, 12 ABT, 13 LUV, 14 RTN, 15 HAL, 16 ATVI, 17 MRK, 18 GPS, 19 GILD, 20 LCC, 21 NKE, 22 MCD, 23 UNH, 24 DOW, 25 M, 26 CBS, 27 COP, 28 CHK, 29 CAT, 30 HON, 31 TWX, 32 AIG, 33 UAL, 34 TXN, 35 BIIB, 36 WAG, 37 PEP, 38 VMW, 39 KO, 40 QCOM, 41 ACN, 42 NOC, 43 DISH, 44 BBY, 45 HD, 46 PG, 47 JNJ, 48 AXP, 49 MAR, 50 TWC, 51 UTX, 52 MA, 53 BLK, 54 EBAY, 55 DAL, 56 NWSA, 57 MSCI, 58 LNKD, 59 TSLA, 60 CVX, 61 AA, 62 NYX, 63 JCP, 64 CMCSA, 65 NDAQ, 66 IT, 67 YHOO, 68 DIS, 69 SBUX, 70 PFE, 71 ORCL, 72 HPQ, 73 S, 74 LMT, 75 XOM, 76 IBM, 77 NFLX, 78 INTC, 79 CSCO, 80 GE, 81 WFC, 82 WMT, 83 AMZN, 84 VOD, 85 DELL, 86 F, 87 TRI, 88 GM, 89 FRT, 90 VZ, 91 FB, 92 BAC, 93 MS, 94 JPM, 95 C, 96 BA, 97 GS, 98 MSFT, 99 GOOG, 100 AAPL. The numerical identifiers are assigned according to the increasing order of the total number of published news in Yahoo! Finance.

We considered three main sources of data for the selected stocks:

\section{Market data}

The first source contains information on price returns and trading volume of the stock at the resolution of the minute. We consider different time scales of investigation, corresponding to $1,10,30,65$, and 130 minutes. The above values are chosen because they are sub-multiple of the trading day in the US markets (from 9:30 AM to 4:00 PM, corresponding to 390 minutes). For each time scale and each stock we extract the following time series:

- $V$, the traded volume in that interval of time,

- $R$, the logarithmic price return in the time scale,

- $\sigma$, the return absolute value, a simple proxy for the stock volatility.

The precise definition of these variables is given in S1 Text. Since trade volumes and absolute price returns are known to display a strong intra-day pattern, we de-seasonalize the corresponding time series (in the same SI Section S1 Text we provide the details about this procedure). This procedure is necessary in order to avoid the detection of spurious correlation and Granger causality due to the presence of a predictable intra-day pattern.

\section{News data}

The second source of data consists of the news published on Yahoo! Finance together with the time series of the aggregated clicks made by the users browsing each page. Yahoo! Finance is a web portal for news and data related to financial companies, offering news and information around stock quotes, stock exchange rates, corporate press releases, financial reports, and message boards for discussion. Providing consumers with a broad range of comprehensive online financial services and information, Yahoo! Finance has consistently been a leader in its category: In May 2008 (see www.comscore.com/Insights/Press-Releases/2008/07/Yahoo!-FinanceTop-Financial-News-and-Research-Site-in-US) it was the top financial website with 18.5 million U.S. visitors, followed by AOL Money \& Finance with 15.2 million visitors (up 48 percent) and MSN Money with 13.7 million visitors (up 13 percent). As of today, recent estimates released in July 2015 (see www.niemanlab.org/2015/07/newsonomics-how-much-is-thefinancial-times-worth-and-who-might-buy-it) confirm that Yahoo! Finance, with more than 72 million visitors, is still the leader finance website in the US, and the fourth in the whole world.

We analyze a portion of the log of Yahoo! Finance, containing news articles displayed on the portal. The articles are tagged with the specific companies (e.g., Google, Yahoo!, Apple, 
Microsoft) or financial entities (e.g., market indexes, commodities, derivatives) that are mentioned in its text. The dataset analyzed in this work does not consist of public data. It was extracted from a browsing log of the Yahoo! Finance web portal. The log stores all the actions made by the users who visit the website, such as views, clicks and comments on every page displayed on the portal. Specifically, we extracted the news articles displayed on Yahoo! Finance and the respective number of "clicks", i.e. the visualizations made by the users. We considered 100 US stocks in a one-year period between 2012 and 2013.

For each considered company we build a signed time series of the sentiment expressed in the related news. The sentiment expressed in each article mentioning a company is weighted by the number of views of the article. In our dataset each click action is associated with a timestamp recording the exact point in time when such action took place. Thus we are able to construct time series at the time resolution of the minute. While building the dataset, we observed the corporate policy of Yahoo with respect to the confidentiality of the data and the tools used in this research. Any sensitive identifier of Yahoo user was discarded after the extraction and aggregation process. Moreover our dataset does not store single actions or users, but only aggregated browsing volumes of financial articles displayed on Yahoo! Finance. Although the original log of Yahoo! Finance is proprietary and cannot obviously be shared, for repeatability of our analysis we can provide the browsing-volume time series extracted for the 100 companies as supplementary material.

In order to automatically detect whether the article is conveying positive or negative news on the company, we perform a sentiment analysis. To obtain a sentiment score, we classify each article with SentiStrength [10], a state-of-the-art tool for extracting positive and negative sentiment from informal texts. The tool is based on a dictionary of "sentiment" words, which are manually picked by expert editors and annotated with a number indicating the amount of positivity or negativity expressed by them. The original dictionary of SentiStrength is not tailored to any specific knowledge or application domain, thus it is not the most proper choice to compute a financial sentiment. To solve this issue, following a practice that is common in most research on sentiment analysis and price returns [11], we adapt the original dictionary by incorporating a list of sentiment keywords of special interest and significance for the financial domain [12]. In S1 Text we discuss the robustness of this choice as well as the way news are associated to stocks.

Supported by previous research that studied stock price reaction to news headlines [13-18], we simplify our data processing pipeline by performing the sentiment analysis computation on the title of each article, instead of using its whole content. The main reason for this choice is that the tone of the news is typically highlighted in the title, while the use in the text of many neutral words can increases the noise and reduces the ability of assessing the sentiment. Finally, the choice also depended on the availability of data: the log at our disposal did not always contained the text of the news and this would have forced us to use a significant subsample.

The sentiment score is a simple sign $(-1,0,+1)$ for each news depending on whether there are more positive or negative words in the title.

\section{Browsing Data}

Finally, in our analysis we use the information on the browsing volume, that is, the time series of "clicks" that the web users made on each article displayed on Yahoo! Finance to view its content. Given that the users' activity on this domain-specific portal proved to provide a clean signal of interest in financial stocks [19], we exploit it in this work to weight the sentiment of each article on a given financial company. Specifically, we use the number of clicks of an article as a proxy for the level of attention that users gave to that news. By aggregating over a time window 
the clicks on all the articles, even published earlier, that mention a particular company, it is possible to derive an estimation of the attention around that company.

In summary, for each time scale and for each stock, the variables we extract from the database are (see S1 Text):

- $C$, the time series of the total number of clicks in a time window,

- $S$, the sum of the sentiment of all news related to each company,

-WS, the sum of the sentiment of all news weighted by the number of clicks.

The first quantity $C$ is non negative and measures the level of attention in a given time interval for news about a specific company. The $S$ variable is the usual sentiment indicator employed in numerous studies and provides the aggregated sentiment of the company specific news published in a given time interval. The most important and novel quantity is WS, which combines the two previous ones by assigning a sign to each click depending on the sentiment of the clicked news. As for the market variables, we remove the intra-day pattern from the click time series. In fact, both the publication of news [16] and the clicking activity of users [19] show a strong intra-day seasonality. These patterns are probably related to the way humans carry out their activities during the day (e.g. small activity during lunchtime, more hectic activity at the beginning or at the end of the business day).

\section{Methods}

\section{Sentiment Analysis}

Regarding the analysis of search-engine queries, some recent works [20-25] have studied the relation between the daily number of queries related to a particular stock and the trading volume over the same stock.

An incomplete list of contributions on the role of news includes studies investigating (i) the relation between exogenous news and price movements [13, 26-28], (ii) the correlation between high or low pessimism of media and high market trading volume [14]; (iii) the relation between the sentiment of news, earnings and return predictability [29, 30], (iv) the role of news in the trading action [16,31-33]; (v) the role of macroeconomic news in the performance of stock returns [34], and (vi) the high-frequency market reaction to news [35].

For example, in a recent paper [21], related to ours, authors show that daily trading volumes of stocks traded at NASDAQ can be forecasted with the daily volumes of queries related to the same stocks. In another paper a similar analysis shows that an increase in queries predicts higher stock prices in the next two weeks [36]. In [25] authors test the explanatory power of investor attention-measured as the search frequency at daily level of stock names in Baidu Index - for abnormal daily returns and find evidence that the former Granger causes stock price returns in excess with respect to the market index return, whereas there is little evidence for the opposite causal relation. As for social networks and micro-blogging platforms [37], Twitter data is becoming an increasingly popular choice for financial forecasting. For example some have investigated whether the daily number of tweets predicts SP 500 stock prices $[38,39]$. A textual analysis approach to Twitter data can be found in other works [15, 40-43] where the authors find clear relations between mood indicators and Dow Jones Industrial Average. Some other authors have used news, Wikipedia data or search trends to predict market movements [26, 44-46].

There are two main critical aspects in the kind of analyses described above. First, the universe of all the search engine or social network users is probably too large and the fraction of users truly interested in finance is likely quite low. This is particularly true at intraday 
frequency, investigated in this paper. Second, as we will empirically show below, the universe of news considered is very heterogeneous in terms of their relevance as a signal of future price movement. For example, in a day there might be several positive but almost irrelevant news and only one negative but very important news on a company. Without weighting the relevance of the news, one could easily draw a wrong conclusion. The intuition behind the current work is that the number of times a news is viewed by users is a measure of its importance as well as of the surprise it conveys. Moreover the users we consider are not generic, but are those who use one of the most important news and search portals for financial information, namely Yahoo! Finance.

\section{Spearman Correlation}

To overcome these limitations we collected for each stock and for each time scale a total of six time series, namely $V, R, \sigma, C, S$, and $W S$, and we study their dependence by making use of two tools. First, given two time series $X_{t}$ and $Y_{t}$, we consider the Spearman's correlation coefficient

$$
\rho(X, Y)=\frac{\left\langle r_{X t} r_{Y t}\right\rangle-\left\langle r_{X t}\right\rangle\left\langle r_{Y t}\right\rangle}{\sqrt{\left(\left\langle r_{X t}^{2}\right\rangle-\left\langle r_{X t}\right\rangle^{2}\right)\left(\left\langle r_{Y_{t}}^{2}\right\rangle-\left\langle r_{Y_{t}}\right\rangle^{2}\right)}}
$$

where $r_{X_{t}}$ and $r_{Y_{t}}$ correspond to the rank of the $t$-th realization of the $X$ and $Y$ random variables, respectively, and $\langle\cdot\rangle$ is the time average value. The correlation $\rho(X, Y)$ quantifies the linear contemporaneous dependence without relying on the Normal assumption for $X$ and $Y$. In order to assess the statistical significance of the measured value we perform a statistical test of the null hypothesis that the correlation is zero by randomizing the time series.

\section{Granger Causality}

Our main goal is testing for the presence of statistical causality between the variables. To this end our second tool is the Granger causality test [47]. Granger's test is a common test used in time series analysis to determine if a time series $X_{t}$ is useful in forecasting another time series $Y_{t} . X_{t}$ is said to Granger-cause $Y_{t}$ if $Y_{t}$ can be better predicted using both the histories of $X_{t}$ and $Y_{t}$ rather than using only the history of $Y_{t}$. The Granger causality can be assessed by regressing $Y_{t}$ on its own time-lagged values and on those of $X_{t}$. An F-test is then used to examine whether the null hypothesis that $Y_{t}$ is not Granger-caused by $X_{t}$ can be rejected with a given confidence level (in this paper we use a p-value of $5 \%$ ).

\section{Results}

The most important aspect of our analysis is to test whether one can forecast financial variables, and more specifically price returns, by using the information on the browsing activity of the users. Namely if, by weighting the sentiment of the clicked news by the number of clicks each news receives, one can improve significantly the predictability of returns.

\section{Heterogenous attention}

The first observation is the extreme heterogeneity of the attention that users of Yahoo! Finance show towards the financial news of a given company. Fig 1 shows the complementary of the cumulative distribution function of the number of clicks per news concerning a given stock. There we show the curves for each of the top 10 stocks and for the aggregate of 100 stocks. In all cases the tail of the distribution is very well fit by a power law behavior [48] with a tail exponent very close to 1 (see S1 Text). In fact, the mean exponent across all stocks is $1.15 \pm 0.30$ and restricting on the top 10 it is $0.99 \pm 0.08$. This indicates that there is a huge heterogeneity in the 


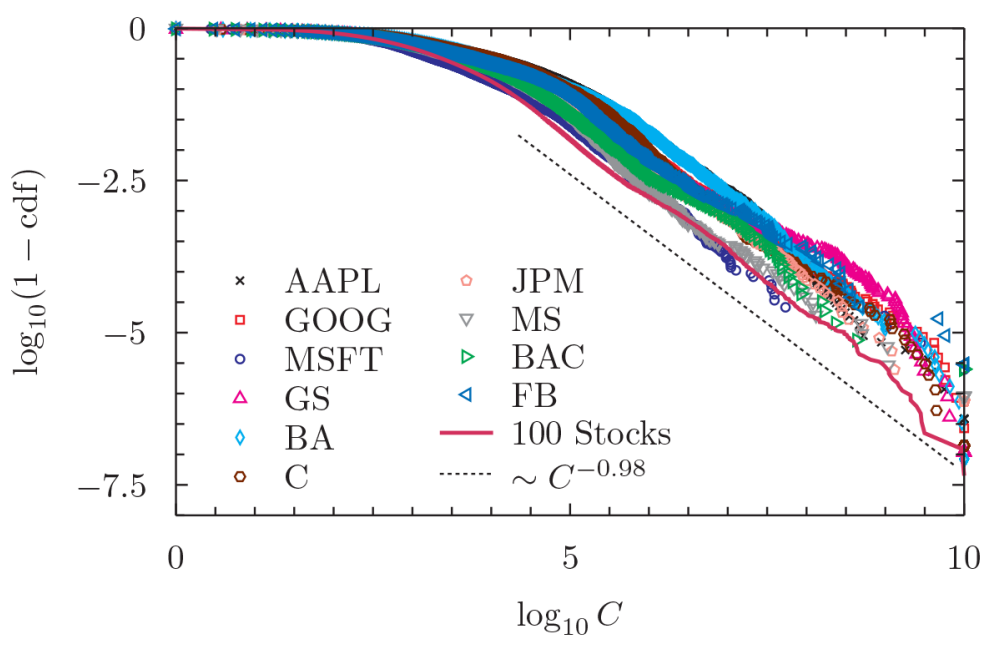

Fig 1. Complementary of the cumulative distribution function of the number of clicks a news receives for the ten assets with the largest number of news and the aggregate portfolio of 100 stocks. Both coordinates have been rescaled by a common factor preserving the power law scaling of the right tail and normalizing the maximum number of clicks to the value $10^{10}$. The dotted line corresponds to a power law with tail exponent fitted from the portfolio time series. We provide details about the standard error and the complete list of tail exponents for all the companies in S1 Text.

doi:10.1371/journal.pone.0146576.g001

number of clicks a news receives and therefore in the importance users give to it. It is also a warning that not weighting properly the importance of the news can lead to overstate the importance of the many irrelevant news and to understate the importance of the few really important ones.

\section{Synchronous correlation}

In order to understand how the relation between financial and news variables depends on the time scale, we perform a synchronous correlation analysis. For each of the 100 companies, we compute the Spearman's correlation coefficient $\rho$ between the three sensible pairs made by one "news" time series and one "financial" time series. Fig 2 summarizes the results for the $65 \mathrm{~min}$ time series. The $\mathrm{x}$ axis lists the companies, uniquely identified by a number that provides the rank of the company in the order from the least to the most cited one (as measured by the absolute number of associated news). Thus, 1 corresponds to the company KBH with the least number of news, while 100 to the most cited AAPL. We label the y axis with the pairs $(C, V),(C, \sigma)$, and $(W S, R)$, while the color scale indicates the level of correlation. We compute the correlation sampling the original time series every 65 minutes, equalizing to zero those values whose significance does not reject the null hypothesis of zero correlation with 5\% confidence. Fig 2 shows in general a positive and significant correlation between browsing activity and price volatility and volume, whereas the evidence of linear dependence between sentiment time series and price returns is mild, similarly to the result obtained by Mao [15]. In the fourth row of Table 1 we report the percentage of the 100 companies for which we reject the null hypothesis of zero correlation at $5 \%$ confidence level and. Since we use multiple correlation tests in order to establish whether their is a significant relationship between key news and online quantities and key market variables, in S1 Text we report results corrected for multiple hypotheses testing. Applying the conservative correction proposed by Bonferroni, the evidence of linear dependence enterily survives for click, volatility, and trade volume time series, whereas the hypothesis of zero-Spearman's correlation between price returns and weighted sentiment is no more rejected. 


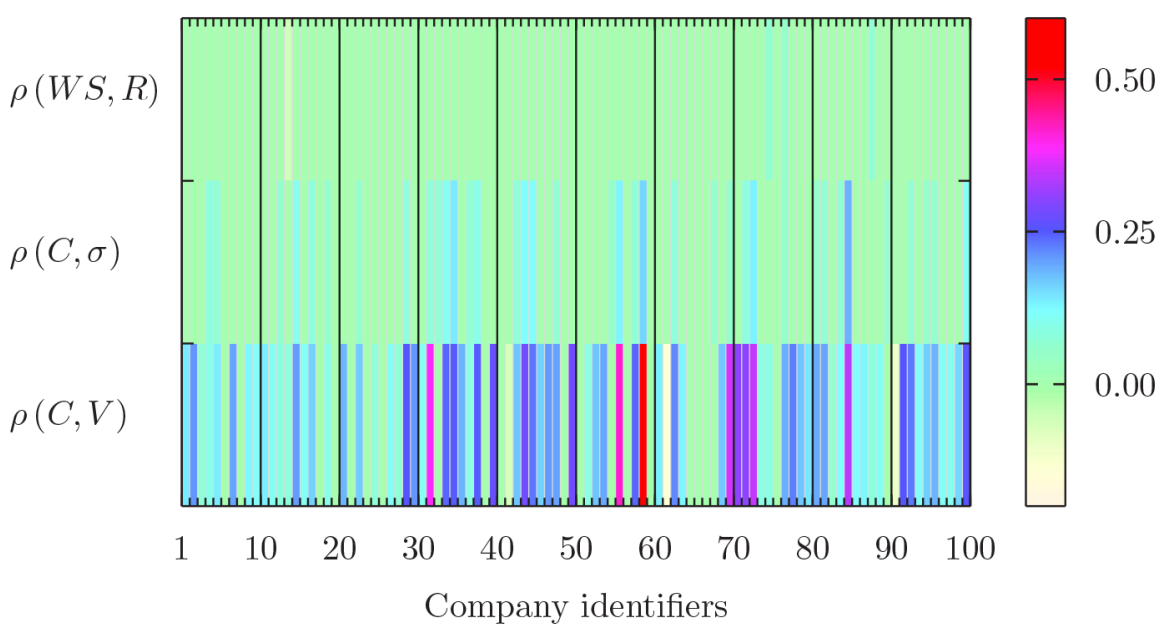

Fig 2. Spearman's correlation coefficients for the de-seasonalized time series of all the $\mathbf{1 0 0}$ companies at hourly scale. The $x$ axis reports the list of companies identified by a unique number, as detailed in the main text. Among the several possibilities, we consider only three couples and the color scale corresponds to the level of correlation. We plot those values for which we reject the null of zero correlation at $5 \%$ significance level and equalize non significant values to zero (light green color).

doi:10.1371/journal.pone.0146576.g002

\section{Time scale}

In order to investigate how the correlation changes with the time scale, in Table 1 we also show the percentage of rejection for the $1,10,30$, and 130 minutes time scales. As a general comment, we observe that the number of companies with a significant correlation becomes higher at finer time resolution. This is a known fact for market variables (e.g. volume and volatility), while we document it for the first time at intraday scale also for browsing variables. The presence of a significant linear relation between the attention given to news articles (signed on the basis of the sentiment expressed in them) related to a given stock and the price return is mild. In particular the low fraction of companies rejecting the null hypothesis is compatible with the expected number of false positives due to multiple testing. Please refer to S1 Text for the detailed results of a multiple test based on the Bonferroni correction.

\section{Dynamics of attention}

The time scale might in principle depend on the relevance of the news. As we have seen, not all news are equal in terms of the attention they receive from the users. To investigate this dependence, we study the dynamics of the number of clicks an article received after its publication. We compute the cumulative number of clicks received by a given news until a given minute after the publication. We perform this for all minutes in a week after the publication. We then normalize

Table 1. Percentage of companies for which we reject the null hypothesis of zero Spearman correlation at $5 \%$ confidence level.

\begin{tabular}{lrrr}
\hline Time interval (minutes) & $\boldsymbol{\rho}(\boldsymbol{W S}, \boldsymbol{R})$ & $\boldsymbol{\rho}(\boldsymbol{C}, \boldsymbol{\sigma})$ & $\boldsymbol{\rho}(\boldsymbol{C}, \boldsymbol{V})$ \\
\hline 1 & 7 & 86 & 95 \\
10 & 3 & 72 & 90 \\
30 & 5 & 54 & 85 \\
65 & 4 & 36 & 79 \\
130 & 4 & 26 & 76 \\
\hline
\end{tabular}

doi:10.1371/journal.pone.0146576.t001 


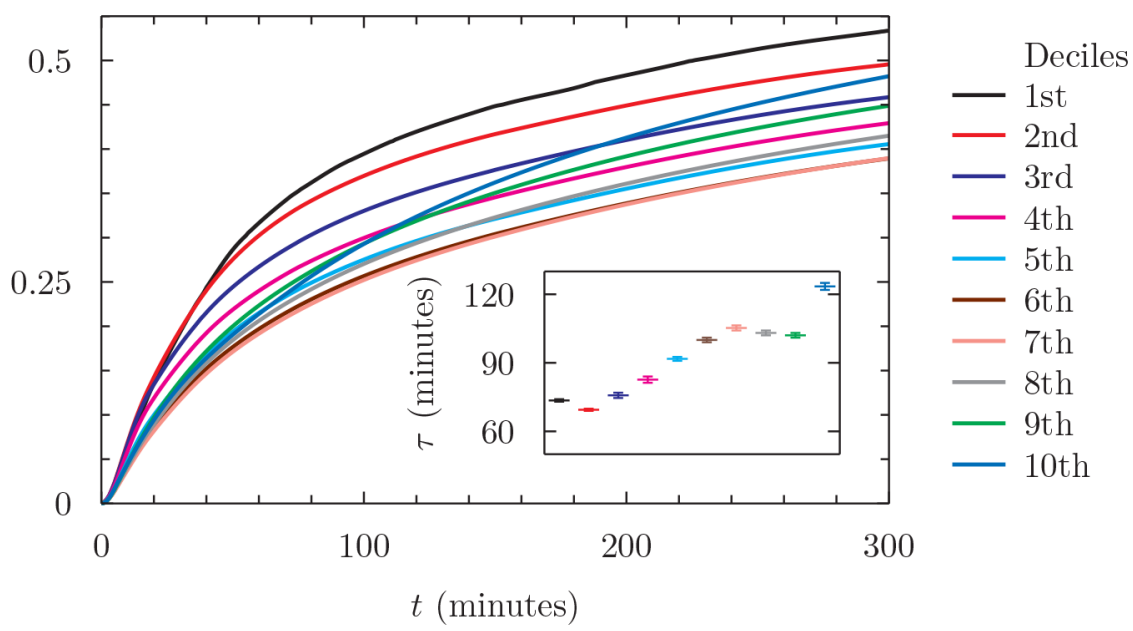

Fig 3. Time evolution of the cumulative number of clicks per news in a time interval of five hours after the publication. We normalize the cumulated amount by a constant which corresponds to the total number of clicks received by a single news during the first week after publication. The news are grouped in deciles according to the total number of clicks they have received until October 2013 and the curves represent average values. Inset: estimated values and standard errors of the attention time scale obtained by an exponential fit of the decile curves.

doi:10.1371/journal.pone.0146576.g003

this cumulative time series by dividing it by the total amount of clicks received by the news. We construct ten groups of news based on the deciles of the total number of news they eventually receive, and we compute for each group the average cumulative sum of clicks. The result is shown in Fig 3. The inset reports for each decile the typical time scale of attention obtained by an exponential fit of the curves. Remarkably, the time scale of attention is an increasing function of the importance of the news (as measured by the total number of clicks). Irrelevant news are immediately recognized as such, while important news continue to receive attention well after their publication. In general, the time scale of the users' attention ranges between one and two hours after the publication, suggesting that this intraday time scale is probably the most appropriate to detect dependencies among financial variables and browsing activity.

\section{Causality}

The synchronous correlation is an important measure of dependence, but not necessarily a sign of causality. Thus we perform a causality analysis by applying Granger's test. We present the results of this analysis, for the 65-minute time horizon, in Fig 4. The $\mathrm{x}$ axis lists the companies as done in Fig 2, while the y axis labels the eight tests that we perform. Black cells correspond to rejection of the null hypothesis of no Granger causality, and the opposite for the white cells. When considering the non-negative variables $(V, C$, and $\sigma)$ we observe strong causal relations. Specifically, in $65 \%$ of the cases the clicking activity causes the trading volume and in $69 \%$ of the cases it causes price volatility. The causality is very strong also in the opposite direction, i.e. volume and volatility cause click volume. This is probably due in part to a reaction of users to anomalously high activity in the market (in terms of volume and/or volatility), while in part it might be a statistical effect due to the fact that all the three variables are very autocorrelated in time, creating strong Granger causal relations in both directions.

We obtain the most interesting and unexpected results when considering the signed variables $(R, S$, and $W S)$. All these variables are weakly serially autocorrelated. When we consider the sentiment of the news ( $S$, without the clicks), we find that only in $4 \%$ of the cases $S$ causes returns, and in $13 \%$ of the cases price return causes $S$. Especially the first value is expected 


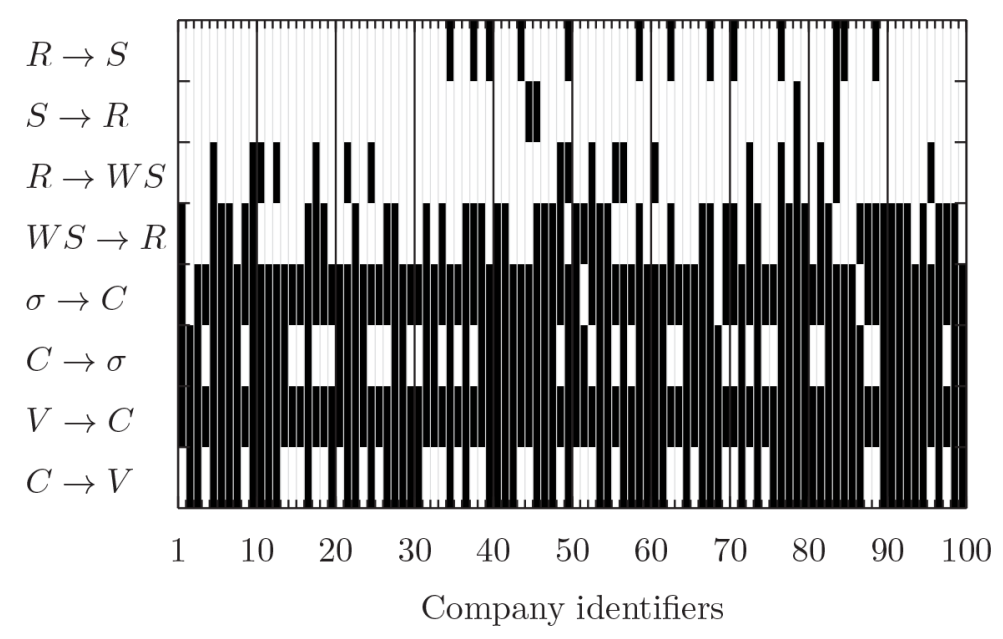

Fig 4. Granger Causality tests at hourly scale between de-seasonalized time series (x axis as in Fig 2). The white cells correspond to tests for which we do not reject the null hypothesis of no Granger causality at $5 \%$ significance level. A black cell corresponds to a statistically significant Granger causality.

doi:10.1371/journal.pone.0146576.g004

under the null, since at $5 \%$ confidence level we expect $5 \%$ of false positive. This means that the simple sentiment of the news does not allow to forecast price returns at intraday (hourly) time scale. On the contrary, when we consider the clicks weighted by the sentiment of the news $(W S)$, we find that in $53 \%$ of the cases it allows predicting returns and only in $19 \%$ of the cases the opposite occurs. In general, companies with more news have higher causality. Our conclusions are even more striking when correcting the test for multiple hypotheses, as done for the Spearman correlation case. The evidence of causality between price returns and unweighted sentiment of the news almost vanishes whereas the signal of causality between weighted sentiment and returns entirely survives. Interestingly, the evidence of causality in the opposite direction-i.e. returns Granger-causing weighted sentiment-weakens and an interesting asymmetric behavior between the two directions clearly emerges. In S1 Text we report the table with detailed results.

\section{Weighting news by users' browsing behavior}

These results show that, on a hourly time scale, the simple news sentiment time series alone (i.e. the one without browsing activity) is not able to predict the price returns; instead, if we add the information provided by the browsing activity, we are then able to properly weigh the news (and its sentiment) by the importance the users give to it by clicking the page. Thus, we find the interesting result that the browsing activity combined with the sentiment analysis of the news increases significantly the level of prediction of price returns for the stocks.

\section{Comparison with existing literature}

Most of the existing studies on sentiment and predictability of returns focus on daily or longer time scale. In order to compare properly our result with the existing literature, we present in Table 2 the results of the above Granger tests on a daily time scale. Table 2 shows that, without the browsing activity, $S$ causes $R$ for $18 \%$ of the companies and $R$ Granger-causes $S$ in $9 \%$ of the cases. Thus there is now some predictability of sentiment, even if the number of companies is quite limited. This is consistent with the existing literature, which reports a weak daily predictability of returns by using sentiment. It is important to note that by adding the browsing 
Table 2. Number of companies for which we reject the null hypothesis of no Granger causality at $5 \%$ confidence level.

\begin{tabular}{lrr}
\hline Causality relation & Hourly scale & Daily scale \\
\hline$S \rightarrow R$ & 4 & 18 \\
$R \rightarrow S$ & 13 & 9 \\
$R \rightarrow W S$ & 19 & 11 \\
$W S \rightarrow R$ & 53 & 37 \\
$V \rightarrow C$ & 100 & 97 \\
$C \rightarrow V$ & 65 & 52 \\
$C \rightarrow \sigma$ & 69 & 52 \\
$\sigma \rightarrow C$ & 96 & 16 \\
\hline
\end{tabular}

doi:10.1371/journal.pone.0146576.t002

activity we can double the number of companies for which there is predictability. In fact, WS Granger-causes $R$ for $37 \%$ of the companies and $11 \%$ in the opposite direction.

\section{Discussion}

The semantic analysis of the news on a specific company is known to have a small predictive power on the future price movements. At the light of our findings, we argue that this effect could be related to the distribution in the attention that the news receive, as clearly emerge in Fig 1: its scale-free behaviour reflects the extreme heterogeneity in the information they convey and the surprise they generate in the readers.

Our in-sample analysis shows that by adding the clicking activity of the web users, we can greatly increase the predictive power of the news for the price returns. This occurs because the time series built with only the sentiment of the news gives the same weight to all the news. In this way even irrelevant news are considered, adding noise to the sentiment time series and reducing the predictive power of the signal. Adding the browsing activity means giving a meaningful weight to each news according to its importance, as measured by the attention it receives by the users, and it enhances the forecast ability of our approach.

The approach to collective evaluation that we proposed in this paper can be useful in many other non financial contexts, since the overflow of information is a common aspect in our lives. In the financial domain, a natural extension of the present work concerns market instabilities and crashes. The analysis presented here is in fact unconditional, i.e. it does not target large price movements or, more generally, abnormal returns. From our societal perspective it would be extremely valuable to have a collective evaluation system, like the one presented here, capable of sifting the relevant information from the pool of data, news, blogs, etc, and to provide early warning indicators of large price movements. Since we have reported evidences in favour of return predictability at intraday time scale-especially at hourly scale-this approach could be also used for real-time indicators, as well as for high-frequency instabilities and systemic price cojumps $[49,50]$, which are becoming increasingly more frequent in our highly automated financial world.

\section{Supporting Information}

S1 Text. Supporting Information for "Coupling news sentiment with web browsing data predicts intra-day stock prices".

(PDF) 


\section{Acknowledgments}

The authors warmly thank Lucio Calcagnile for the valuable technical support during the final stage of this work. The opinions expressed here are solely those of the authors and do not represent in any way those of their employers.

\section{Author Contributions}

Conceived and designed the experiments: GR IB GB GC FL MT. Performed the experiments: GR IB GB GC FL MT. Analyzed the data: GR IB GB GC FL MT. Contributed reagents/materials/analysis tools: GR IB GB GC FL MT. Wrote the paper: GR IB GB GC FL MT.

\section{References}

1. King G. Ensuring the data-rich future of the social sciences. Science 331,719-721 (2011). doi: 10. 1126/science.1197872 PMID: 21311013

2. Vespignani A. Predicting the behavior of techno-social systems. Science $325,425-428$ (2009). doi: 10.1126/science.1171990 PMID: 19628859

3. Bonanno G. et al. Networks of equities in financial markets. Eur. Phys. J. 38, 363-371 (2004). doi: 10. 1140/epjb/e2004-00129-6

4. Caldarelli G. Scale-Free Networks: complex webs in nature and technology ( Oxford University Press, Oxford, 2007).

5. Tumminello M., Aste T., Di Matteo T. \& Mantegna R. N. A tool for filtering information in complex systems. P.N.A.S. 102, 10421-10426 (2005). doi: 10.1073/pnas.0500298102 PMID: 16027373

6. Achrekar, H., Gandhe, A., Lazarus, R., Yu, S.-H. \& Liu, B. Predicting flu trends using twitter data. In Computer Communications Workshops (INFOCOM WKSHPS), 2011 IEEE Conference on, 702-707 (IEEE, 2011).

7. Tizzoni M. et al. Real-time numerical forecast of global epidemic spreading: case study of $2009 \mathrm{~A} /$ H1N1pdm. BMC medicine 10, 165 (2012). doi: 10.1186/1741-7015-10-165 PMID: 23237460

8. Caldarelli G. et al. A multi-level geographical study of Italian political elections from Twitter data. PloS one 9, e95809 (2014). doi: 10.1371/journal.pone.0095809 PMID: 24802857

9. Malkiel B. G. \& Fama E. F. Efficient capital markets: A review of theory and empirical work. J. Finance 25, 383-417 (1970). doi: 10.2307/2325486

10. Thelwall M., Buckley K., Paltoglou G., Cai D. \& Kappas A. Sentiment strength detection in short informal text. J. Am. Soc. Inf. Sci. Technol. 61, 2544-2558 (2010). doi: 10.1002/asi.21416

11. Wang, C., Tsai, M., Liu, T., \& Chang, C. Financial Sentiment Analysis for Risk Prediction. In Proceedings of the Sixth International Joint Conference on Natural Language Processing, 802-808, (2013).

12. Loughran T. \& McDonald B. When is a liability not a liability? Textual analysis, dictionaries, and 10-Ks. J. Finance 66, 35-65 (2011). doi: 10.1111/j.1540-6261.2010.01625.x

13. Chan W. S. Stock price reaction to news and no-news: drift and reversal after headlines. J. Fin. Econ. 70, 223-260 (2003). doi: 10.1016/S0304-405X(03)00146-6

14. Tetlock P. C. Giving content to investor sentiment: The role of media in the stock market. J. Finance 62 , 1139-1168 (2007). doi: 10.1111/j.1540-6261.2007.01232.x

15. Mao, H., Counts, S. \& Bollen, J. Predicting financial markets: Comparing survey, news, Twitter and search engine data. Preprint arXiv:1112.1051 (2011).

16. Lillo F., Miccichè S., Tumminello M., Piilo J. \& Mantegna R. N. How news affect the trading behavior of different categories of investors in a financial market. Quant. Finance 15, 213-229 (2015). doi: 10. 1080/14697688.2014.931593

17. Reis, J. C., Benvenuto, F., Vaz de Melo, P., Prates, O., Kwak, H., \& An, J. Breaking the News: First Impressions Matter on Online News In ICWSM'15: Proceedings of The International Conference on Weblogs and Social Media, 2015, (2015).

18. Ruiz-Martinez, J.M., Valencia-Garcia, R., \& Garcia-Sanchez, F. Semantic-Based Sentiment analysis in financial news. In Proceedings of the First International Workshop on Finance and Economics on the Semantic Web (FEOSW 2012) in conjunction with 9th Extended Semantic Web Conference (ESWC 2012), (2012).

19. Bordino, I., Kourtellis, N., Laptev, N. Billawala, Y. Stock trade volume prediction with yahoo finance user browsing behavior. In Data Engineering (ICDE), 2014 IEEE 30th International Conference on, 1168-1173 (2014). 
20. Bank M., Larch M. \& Peter G. Google search volume and its influence on liquidity and returns of german stocks. Fin. Mar. Port. Man. 25, 239-264 (2011). doi: 10.1007/s11408-011-0165-y

21. Bordino I.et al. Web search queries can predict stock market volumes. PloS One 7, e40014 (2012). doi: 10.1371/journal.pone.0040014 PMID: 22829871

22. Preis T., Reith D. \& Stanley H. E. Complex dynamics of our economic life on different scales: insights from search engine query data. Philos. T. R. Soc. A. 368, 5707-5719 (2010). doi: 10.1098/rsta.2010. 0284

23. Kristoufek L. Can Google Trends search queries contribute to risk diversification? Sci.Rep. 3 (2013). doi: 10.1038/srep02713

24. Vlastakis N. \& Markellos R. N. Information demand and stock market volatility. J. Ban. Fin. 36, 18081821 (2012). doi: 10.1016/j.jbankfin.2012.02.007

25. Zhang W., Shen D., Zhang Y. \& Xiong X. Open source information, investor attention, and asset pricing. Economic Modelling 33, 613-619 (2013). doi: 10.1016/j.econmod.2013.03.018

26. Curme C., Preis T., Stanley H. E. \& Moat H. S. Quantifying the semantics of search behavior before stock market moves. P.N.A.S. 111, 11600-11605 (2014). doi: 10.1073/pnas.1324054111 PMID: 25071193

27. Cutler D. M., Poterba J. M. \& Summers L. H. What moves stock prices? J. Port. Man. 15, 4-12 (1989). doi: $10.3905 / j p m .1989 .409212$

28. Vega C. Stock price reaction to public and private information. J. Fin. Econ. 82, 103-133 (2006). doi: 10.1016/j.jfineco.2005.07.011

29. Tetlock P. C., Saar-Tsechansky M. \& Macskassy S. More than words: Quantifying language to measure firms' fundamentals. J. Finance 63, 1437-1467 (2008). doi: 10.1111/j.1540-6261.2008.01362.x

30. Schumaker R. P. \& Chen H. Textual analysis of stock market prediction using breaking financial news: The AZFin text system. ACM (TOIS) 27, 12 (2009).

31. Engelberg J. E., Reed A. V. \& Ringgenberg M. C. How are shorts informed? Short sellers, news, and information processing. J. Fin. Econ. 105, 260-278 (2012). doi: 10.1016/j.jfineco.2012.03.001

32. Alanyali M., Moat H. S. \& Preis T. Quantifying the relationship between financial news and the stock market. Sci. Rep. 3 (2013). doi: 10.1038/srep03578 PMID: 24356666

33. Zhang Y. et al. Internet information arrival and volatility of sme price index. Physica $A$ 399, 70-74 (2014). doi: 10.1016/j.physa.2013.12.034

34. Birz G. \& Lott J. R Jr. The effect of macroeconomic news on stock returns: New evidence from newspaper coverage. J. Ban. Fin. 35, 2791-2800 (2011). doi: 10.1016/j.jbankfin.2011.03.006

35. Gross-Klussmann A. \& Hautsch N. When machines read the news: Using automated text analytics to quantify high frequency news-implied market reactions. J. Emp. Fin. 18, 321-340 (2011). doi: 10.1016/ j.jempfin.2010.11.009

36. Da Z., Engelberg J. \& Gao P. In search of attention. J. Finance 66, 1461-1499 (2011). doi: 10.1111/j. 1540-6261.2011.01679.x

37. De Choudhury, M., Sundaram, H., John, A. \& Seligmann, D. D. Can blog communication dynamics be correlated with stock market activity? In Proceedings of the nineteenth ACM conference on Hypertext and hypermedia, 55-60 (ACM, 2008).

38. Mao, Y., Wei, W., Wang, B. \& Liu, B. Correlating S\&P 500 stocks with Twitter data. In Proceedings of the First ACM International Workshop on Hot Topics on Interdisciplinary Social Networks Research, 69-72 (ACM, 2012).

39. Ruiz, E. J., Hristidis, V., Castillo, C., Gionis, A. \& Jaimes, A. Correlating financial time series with microblogging activity. In Proceedings of the fifth ACM international conference on Web search and data mining, 513-522 (ACM, 2012).

40. Bollen J., Mao H. \& Zeng X. Twitter mood predicts the stock market. J. Comp. Sci. 2, 1-8 (2011). doi: 10.1016/j.jocs.2010.12.007

41. Bollen, J., Pepe, A. \& Mao, H. Modeling public mood and emotion: Twitter sentiment and socio-economic phenomena. In Proceedings of the Fifth International AAAI Conference on Weblogs and Social Media, 450-453 (2011).

42. Zhang X., Fuehres H. \& Gloor P. A. Predicting stock market indicators through Twitter "I hope it is not as bad as I fear". Proc. Soc. Beh. Sci. 26, 55-62 (2011). doi: 10.1016/j.sbspro.2011.10.562

43. Zheludev I., Smith R. \& Aste T. When can social media lead financial markets?. Sci. Rep. 4 (2014). doi: 10.1038/srep04213

44. Preis T., Moat H. S., Stanley H. E. \& Bishop S. R. Quantifying the advantage of looking forward. Sci. Rep. 2 (2012). doi: 10.1038/srep00350 
45. Preis T., Moat H. S. \& Stanley H. E. Quantifying trading behavior in financial markets using Google Trends. Sci. Rep. 3 (2013). doi: 10.1038/srep01684

46. Moat H. S. et al. Quantifying wikipedia usage patterns before stock market moves. Sci. Rep. 3 (2013). doi: 10.1038/srep01801

47. Granger C. Investigating causal relations by econometric models and cross-spectral methods. Econometrica 37, 424-438 (1969). doi: 10.2307/1912791

48. Zipf G. Human Behavior and the Principle of Least Effort ( Addison-Wesley, Cambridge, MA, 1949).

49. Bormetti G. et al. Modelling systemic price cojumps with Hawkes factor models. Quant. Finance 15, 1137-1156 (2015). doi: 10.1080/14697688.2014.996586

50. Calcagnile, L. M. et al. Collective synchronization and high frequency systemic instabilities in financial markets. Preprint arXiv:1505.00704 (2015). 